\title{
Logrolling and the Paradox of Voting: Are They Really Logically Equivalent? A Comment
}

\author{
Peter Bernholz \\ Universität Basel, Switzerland
}

In his paper "Vote-Trading and the Voting Paradox: A Proof of Logical Equivalence" David Koehler ${ }^{1}$ claims to have proved the equivalence of logrolling and the paradox of voting. Unfortunately, the proofs given by Koehler do not have the general validity he intends them to have. Indeed, it is easy to show that logrolling does not necessarily imply the paradox of voting, nor does the paradox of voting imply the existence of logrolling situations.

I begin by first demonstrating with the help of a counter-example that certain logrolling situations do not imply the paradox of voting. This is not very surprising, since to prove that logrolling implies the paradox, it has been necessary to assume the absence of a certain kind of complementarity among issues. ${ }^{2}$ In the counter-example a group of three voters, $V_{1}, V_{2}$ and $V_{3}$, has to decide two issues $x$ and $y$. The two issues each contain two alternatives, namely, $x_{1}, x_{2}$ and $y_{1}$, $y_{2}$. In Matrix 1 the utility payoffs for voters $V_{1}$, $V_{2}$ and $V_{3}$ are given for the four possible outcomes $\left(x_{1}, y_{1}\right),\left(x_{1}, y_{2}\right),\left(x_{2}, y_{1}\right)$ and $\left(x_{2}, y_{2}\right)$.

\section{Matrix 1}

\begin{tabular}{c|c|c}
\hline Moves available to all voters & $x_{1}$ & $x_{2}$ \\
\hline$y_{1}$ & $8,8,8$ & $2,10,2$ \\
\hline$y_{2}$ & $6,2,10$ & $10,6,6$ \\
\hline
\end{tabular}

With simple majority voting $x_{2}$ and $y_{2}$ will receive a majority of votes, so that $\left(x_{2}, y_{2}\right)$ will be the outcome selected by the group. This result follows, because $x_{1}$ is dominated by $x_{2}$ for $V_{2}$, and $y_{1}$ by $y_{2}$ for $V_{3}$. With no knowledge about the behavior of the other voters $V_{2}$ will, therefore, vote for $x_{2}$ and $V_{3}$ for $y_{2} . V_{1}$ knows this situation and consequently casts his votes for $x_{2}$ and $y_{2}$, since by doing so his most preferred outcome $\left(x_{2}, y_{2}\right)$ will get a majority.

'David H. Koehler, "Vote-Trading and the Voting Paradox: A Proof of Logical Equivalence," American Political Science Review, 69 (September, 1975), 954960.

${ }^{2}$ Peter Bernholz, "Logrolling, Arrow-Paradox and Decision Rules: A Generalization," Kyklos, 22 (fasc. 1, 1974), 53, assumption A5.
But now voters $V_{2}$ and $V_{3}$ prefer $\left(x_{1}, y_{1}\right)$ to $\left(x_{2}, y_{2}\right)$ because of the higher payoffs $8>6$. And they can obtain this outcome by making a logrolling agreement. According to this agreement $V_{2}$ votes for $x_{1}$ as against $x_{2}$, which is against his immediate interest, to get in exchange $V_{3}$ 's vote for $y_{1}$ as against $y_{2}$, which is contrary to $V_{3}$ 's immediate advantage. This exchange of votes is, however, favorable to both, since $y_{1}$ strongly dominates $y_{2}$ for $V_{2}$, and $x_{1}$ strongly dominates $x_{2}$ for $V_{3}$. Consequently, a typical logrolling situation is present.

It is now quite obvious that in the case given above, logrolling does not imply the paradox of voting. For $\left(x_{2}, y_{1}\right)$ as well as $\left(x_{1}, y_{2}\right)$ are preferred to $\left(x_{1}, y_{1}\right)$ by only one voter. $\left(x_{1}, y_{1}\right)$ is a stable outcome. Note, moreover, that $\left(x_{2}, y_{2}\right) P_{1}\left(x_{2}, y_{1}\right)$, $\left(x_{2}, y_{2}\right) P_{1}\left(x_{1}, y_{2}\right)$ and $\left(x_{1}, y_{1}\right) P_{1}\left(x_{2}, y_{1}\right),\left(x_{1}, y_{1}\right)$ $P_{1}\left(x_{1}, y_{2}\right)$, where $P_{1}$ means "preferred to by $V_{1}$." As a consequence just the kind of complementarity is present, the absence of which had to be assumed to prove that logrolling situations imply the paradox of voting. ${ }^{2}$

I proceed to show that cyclical group preferences do not generally imply the presence of logrolling situations. First, it has to be mentioned that Koehler has not given a proof for cycles containing more than three alternatives. But it is obvious that cyclical group preference orderings with more than three alternatives $A_{1}$ can exist. In general, we have:

$$
A_{1} P A_{2} P A_{3} P \cdots P A_{n-1} P A_{n} P A_{1},
$$

where $P$ means "preferred to by a majority of the group." Koehler's proof is only concerned with the case, in which $n=3$, and no proof has been given for $n>3$.

Secondly, consider Koehler's case with $n=3$ :

$$
A_{1} P A_{2} P A_{3} P A_{1} \text {. }
$$

Even in this case the paradox of voting need not imply logrolling situations. For assume that $A_{1}, A_{2}$ and $A_{3}$ belong all to one issue $A$. For example, the $A_{i}$ may represent three different bridges, out of which one has to be selected by the group, since only one bridge can be built because of budgetary 
restrictions. In this case substantive logrolling is obviously not possible, since there are no two issues concerning which votes can be exchanged by group members.

The above considerations prove not only that Koehler has failed to show the logical equivalence of logrolling and the paradox of voting but also that such a proof is impossible. What Koehler has demonstrated-but only for three group members and for two issues-is that under certain conditions logrolling and the paradox of voting are equivalent. But his belief that he had given a general proof prevented him from trying to find these conditions. As mentioned above, one of these conditions is the absence of a certain kind of complementarity among the relevant issues. 